\title{
The high level trigger for rare decays at $\mathrm{LHCb}$
}

\author{
Kim Vervink ${ }^{* \dagger}$ \\ CERN \\ E-mail: kim.vervink@cern.ch
}

The LHCb experiment is dedicated to the study of $C P$ violation and rare $B$ decays. A trigger system is developed to filter these decays from the LHC background environment. An overview of the LHCb trigger strategy is given in this paper as well as an illustration on how the trigger selects the rare decays $B_{d} \rightarrow K^{* 0} \mu^{+} \mu^{-}$and $B_{s} \rightarrow \phi \gamma$ and their control samples, which are used to parametrize possible biases.

The 2009 Europhysics Conference on High Energy Physics,

July 16 - 222009

Krakow, Poland

*Speaker.

${ }^{\dagger}$ On behalf of the LHCb Collaboration. 


\section{Introduction}

Present constraints on physics beyond the Standard Model (SM) are heavily influenced by measurements of rare processes where observables are theoretically well controlled. Rare $B$ decays will offer opportunities to make further world class constraints on new physics at the LHC, and a host of such decays will be studied at the LHCb experiment. Given the small cross section of very rare decays in question, the trigger is a critical aspect for isolating such decays in the LHC experimental environment. At LHCb the trigger will consist of a first level hardware trigger (L0) and a second 'High Level Trigger' (HLT), based on software algorithms running in a CPU-farm. The trigger is required to reduce the rate to $2 \mathrm{kHz}$, which is allowed by the long-term data storage system. Meanwhile it needs to provide a good efficiency on interesting channels, such as the rare $B_{d} \rightarrow K^{* 0} \mu^{+} \mu^{-}$or $B_{s} \rightarrow \phi \gamma$ decays as well as their control channels, which are used to establish efficiencies and to provide calibration samples.

\section{The LHCb trigger}

The bunch crossing rate at $\mathrm{LHC}$ is $40 \mathrm{MHz}$, but the rate at $\mathrm{LHCb}$ when both bunches are filled, is $30 \mathrm{MHz}$. However only $10 \mathrm{kHz}$ of crossings have at least one $B$ meson decaying in the detector acceptance at the nominal luminosity of $2 \times 10^{32} \mathrm{~cm}^{2} \mathrm{~s}^{-1}$. The two rare decays discussed in the next section have a production rate much less than a Hz. A dedicated trigger system [1] is therefore essential to filter these events from the QCD background.

\subsection{The L0 hardware trigger}

The purpose of the $\mathrm{L} 0$ trigger is to reduce the rate to $1 \mathrm{MHz}$ within a $4 \mu$ s latency. Therefore only part of the $\mathrm{LHCb}$ detector is read out at this stage: the Pile-Up system, the calorimeters and the muon stations.

The L0 muon trigger selects the two muon tracks with the highest $p_{\Gamma}$ in each quadrant of the muon stations. A L0 muon trigger is issued if the candidate with the highest $p_{\Gamma}$ or if the sum of the two highest $p_{\mathrm{T}}$ candidates is above a certain threshold.

The L0 calorimeter trigger reconstructs and selects particles with a high transverse energy deposit in the calorimeters. It provides candidates for hadrons, electrons, photons and neutral pions. The properties of the showers together with the SPD ${ }^{1}$ and Pre-Shower detector information provide a first particle hypothesis. An event can be triggered if the transverse energy of a cluster is above a certain threshold. A possible value for the early data taking could be $E_{\mathrm{T}}>860 \mathrm{MeV}$ for an hadronic cluster.

\subsection{The HLT trigger}

The High Level Trigger (HLT) is an application running in the Event Filter Farm on L0 accepted events. The detector is fully read out at a rate of $1 \mathrm{MHz}$. The HLT is divided into two levels, as illustrated in Figure 1. The first level (HLT1) applies different sequences of algorithms (called alleys) depending on the decision issued by the L0 trigger and reduces the output rate to a few tens

\footnotetext{
${ }^{1}$ Scintillating Pad Detector with cells matching the Electromagnetic Calorimeter cells.
} 
of $\mathrm{kHz}$. The HLT1 requires a confirmation of the corresponding L0 candidates by adding information from either the VELO or the main tracker and applies cuts on the transverse momentum and the impact parameter (IP) with respect to the primary vertex. For some alleys, additional particles apart from the $\mathrm{L} 0$ candidate are searched for to give a better discrimination between $B$-decay products and background.

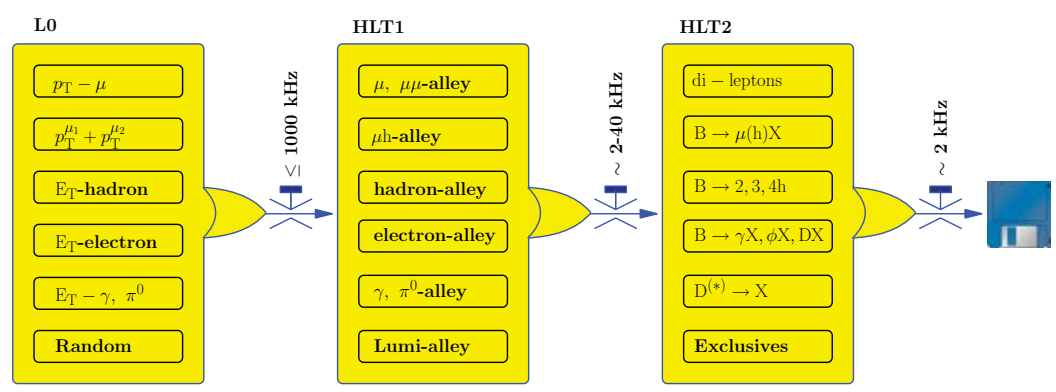

Figure 1: Overview of the LHCb trigger scheme.

The second level (HLT2) consists of inclusive selections as well as a handful of exclusive selection algorithms. The full offline pattern recognition is engaged on the HLT1 selected events and cuts are applied on robust quantities such as the momentum and the IP. The tracks of the remaining candidates are then Kalman fitted, which provides us not only with better track estimates but also with track errors, such that a selection can be applied on the IP significance, vertex and track quality. At this stage some alleys even run a fast particle identification algorithm to further discriminate background. The HLT2 reduces the rate further to $2 \mathrm{kHz}$ where it is sent to storage.

\section{Rare decays through the trigger}

\section{1 $B_{d} \rightarrow K^{* 0} \mu \mu$}

The electroweak $B_{d} \rightarrow K^{* 0} \mu \mu$ decay proceeds mostly via a penguin diagram with a branching fraction, $\mathscr{B} \sim 1.2 \times 10^{-6}$. In a range of new physics models, the angular distribution of the muons can differ significantly from the SM. One of the most promising observables is the difference in the forward-backward asymmetry $\left(A_{\mathrm{FB}}\right)$ of the muons as a function of the dimuon mass, $q^{2}$. In particular, the value of the dimuon mass at which the asymmetry is zero is predicted to be $4.36_{-0.31}^{+0.33} \mathrm{GeV} / \mathrm{c}^{2}$ in the SM [2].

This channel is triggered mainly through the L0 single and dimuon triggers and by the muon and the muon-plus-track alley in the HLT. The overall trigger efficiency on offline selected $B_{d} \rightarrow$ $K^{* 0} \mu \mu$ events is $\sim 80 \%$ [3].

A cut applied on both muons $p_{T}$ 's in the trigger algorithms results in the events that are most sensitive in $A_{\mathrm{FB}}$ to be removed. A control sample, $B_{d} \rightarrow J / \psi K^{0 *}$, is triggered and selected by the same filters as the signal decay and is produced with a well known angular distribution such that it can be used to parametrize the angular acceptance function of the signal.

Simulation studies have shown that the expected number of signal events in $2 \mathrm{fb}^{-1}$ is $\sim 4300$, which allows the zero-crossing point to be measured with a precision of $\pm 0.5 \mathrm{GeV}$ [4]. 


\section{2 $B_{s}^{0} \rightarrow \phi \gamma$}

The $B_{s}^{0} \rightarrow \phi \gamma$ decay is a radiative process described in the SM by a $b \rightarrow s \gamma$ penguin diagram and its measured branching ratio is $\mathscr{B} \sim 5.7 \times 10^{-5}$ [5]. The radiative photon is expected to be left handed polarized with an admixture of right-handed component at the level of $\mathscr{O}\left(\mathrm{m}_{\mathrm{b}} / \mathrm{m}_{b}\right)$. However in several extensions of the SM, the photon can acquire an appreciable right-handed component $[6,7]$. From the time-dependent decay rate of $B_{s}\left(\bar{B}_{s}\right)$ to $\phi \gamma$, one can measure the coefficient $A^{\Delta}$, which is directly related to the polarization of the radiative photon [8].

$$
\left.\Gamma \stackrel{(-)}{B_{s}^{0} \rightarrow \phi \gamma}\right)=|A|^{2} e^{-\Gamma_{s} t}\left[\cosh \left(\frac{\Delta \Gamma_{s} t}{2}\right)+A^{\Delta} \sinh \left(\frac{\Delta \Gamma_{s} t}{2}\right) \pm C \cos \left(\Delta m_{s} t\right) \mp S \sin \left(\Delta m_{s} t\right)\right]
$$

$B_{s}^{0} \rightarrow \phi \gamma$ is mainly triggered by the radiative L0 trigger and is confirmed in HLT1 by a trigger searching for a photon and a track signal. In HLT2 an inclusive $\phi$ trigger and a dedicated exclusive trigger yield a 95\% efficiency on the HLT1 accepted events. The overall trigger efficiency for offline selected $B_{s}^{0} \rightarrow \phi \gamma$ events is of the order of $\sim 60 \%$. An HLT cut on the IP of the $B_{s}$ introduces a bias in the lifetime distribution which can be parametrized using the control sample $b_{s}^{0} \rightarrow K^{*}\left(K^{+} \pi^{-}\right) \gamma$. This decay is triggered by the same selection but produced with 6 times more statistics.

There are $\sim 11 \cdot 10^{3}$ signal events expected in $2 \mathrm{fb}^{-1}$ of accumulated statistics. This corresponds to a statistical uncertainty on $C$ and $S$ of $\sim 0.1$ and on $A^{\wedge}$ of approximately $\sim 0.2 \%$ [9].

\section{Conclusion}

The current strategy of the LHCb trigger system has been presented in this paper. The L0 hardware trigger and the HLT software trigger have proved to be successful on simulated data in reducing the background to the acceptable rate, while keeping the efficiency high for the key channels. We are waiting eagerly to determine the performance using data from real collisions. Two important rare decays, $B_{d} \rightarrow K^{* 0} \mu^{+} \mu^{-}$and $B_{s} \rightarrow \phi \gamma$ have been introduced. Both channels have a high trigger efficiency but also their control sample is selected through the same trigger lines. These control samples will be used to parametrize any induced biases.

\section{References}

[1] The LHCb Collaboration, LHCb Trigger System Technical Design Report, CERN/LHCC 03-031.

[2] M. Beneke, Th. Feldmann, D. Seidel, Eur. Phys. J C 41 (2005) 173, arXiv:hep-ph/0412400.

[3] The LHCb Collaboration, Roadmap for the analysis $B_{s} \rightarrow K^{0 *} J / \psi(\mu \mu)$, in preparation, LHCb-ROADMAP2-001.

[4] J. Dickens, V. Gibson, C. Lazzeroni, M. Patel, LHCb-2007-038.

[5] J. Wicht et al. Belle Collaboration, Phys. Rev. Lett 100 (2008) 121801.

[6] R. N. Mohapatra and J. C. Pati, Phys. Rev. D11 (1975) 566; G. Senjanovic and R. N. Mohapatra, Phys. Rev. D12 (1975) 1502; G. Senjanovic, Nucl. Phys. B153 (1979) 334.

[7] H. E. Haber and G. L. Kane, Phys. Rept.117 (1985) 75.

[8] D. Atwood, M. Gronay and A. Soni, Phys. Rev. Lett.(1997) 15 [arXiv:hep-ph/9704272].

[9] L. Shchutska, Y. Xie, A. Golutvin, V. Egorychev, V. Shevchenko, I. Belyaev, LHCb-PHYS-2007-147. 